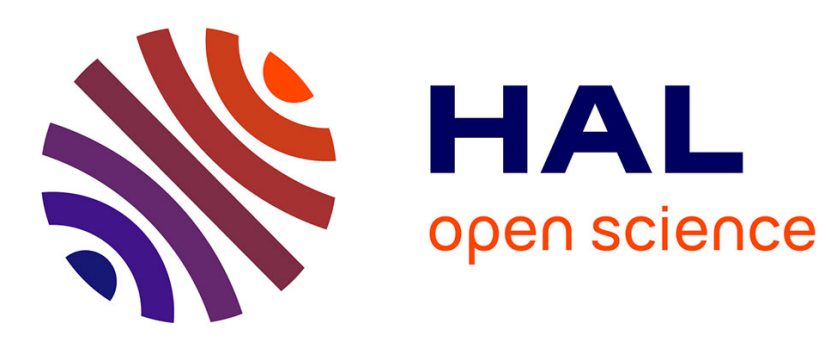

\title{
Homogenization of 3D finite chiral photonic crystals
}

Sébastien Guenneau, Frédéric Zolla

\section{To cite this version:}

Sébastien Guenneau, Frédéric Zolla. Homogenization of 3D finite chiral photonic crystals. Physica B:

Condensed Matter, 2007, 394 (2), pp.145-147. hal-00186679

\section{HAL Id: hal-00186679 \\ https://hal.science/hal-00186679}

Submitted on 3 Dec 2007

HAL is a multi-disciplinary open access archive for the deposit and dissemination of scientific research documents, whether they are published or not. The documents may come from teaching and research institutions in France or abroad, or from public or private research centers.
L'archive ouverte pluridisciplinaire HAL, est destinée au dépôt et à la diffusion de documents scientifiques de niveau recherche, publiés ou non, émanant des établissements d'enseignement et de recherche français ou étrangers, des laboratoires publics ou privés. 


\title{
Homogenization of 3D finite chiral photonic crystals
}

\author{
S. Guenneau ${ }^{\mathrm{a}, \mathrm{b}}$ F. Zolla ${ }^{\mathrm{a}}$ \\ anstitut Fresnel, UMR CNRS 6133, University Aix-Marseille I, case 162, F13397 Marseille Cedex 20, France \\ b Department of Mathematical Sciences, $M$ \& $O$ Building, \\ Peach Street, Liverpool L69 3BX, United Kingdom University
}

\begin{abstract}
We homogenize Maxwell's equations for Drude-Born-Fedorov chiral crystals, using the multi-scale technique. We find that the homogenized material is described by dispersive anisotropic effective matrices, derived from frequency dependent coupled auxiliary problems. For low frequencies, these matrices may not be positive definite.
\end{abstract}

Key words: Multi-scale method, Homogenization, Meta-materials PACS:

\section{Introduction}

Between 1996 and 1999, the British physicist John Pendry published a series of papers giving rise to a new class of composite structures (christened metamaterials) which exhibit low plasmon frequency (arrays of thin straight wires) and artificial magnetism (split ring resonators) in the low frequency regime (homogenization domain). When combined, these two structures may exhibit negative refractive index as was chiefly demonstrated by David Smith and others in the microwave regime. Recently, John Pendry proposed a new route to negative refraction through arrays of chiral inclusions of helicoidal shape [1]: Backward waves can propagate in composite structures with positive optical parameters provided that one of the constituent materials is chiral. This may open new vistas for negative refraction in the optical region via homogenized chiral media (the characteristic dimensions in the composite structure are much smaller than the wavelength). This is the main motivation for the present analysis of effective properties of finite periodic chiral structures.

\section{Homogenization setting}

In this paper, we consider the diffraction problem of a monochromatic wave incident on a heterogeneous chiral body, when its wavelength is large compared to the typical heterogeneity size, but possibly in resonance with the overall finite structure. To model this, let us introduce a unit cell $Y=\left[0 ; 1\left[^{3}\right.\right.$ whose homothety $\eta Y$ is repeated periodically within a fixed bounded domain $\Omega$. When $\eta$ tends to zero, $\Omega$ is filled by with an increasing number of very tiny cells, so that it looks homogeneous. We also define the relative permittivity and permeability and the chiral inductance at every point $\mathbf{x} \in \mathbb{R}^{3}$ by $\chi_{\eta}(\mathbf{x})=\tilde{\chi}\left(\mathbf{x}, \frac{\mathbf{x}}{\eta}\right), \chi \in\left\{\varepsilon_{r}, \mu_{r}, \beta\right\}$ with

$$
\tilde{\chi}(\mathbf{x}, \mathbf{y})= \begin{cases}\chi(\mathbf{y}) & , \text { if } \mathbf{x} \in \Omega, \\ 1 & \text { for } \chi=\varepsilon_{r}, \mu_{r}, \text { if } \mathbf{x} \in \mathbb{R}^{3} \backslash \bar{\Omega}, \\ 0 & \text { for } \chi=\beta, \text { if } \mathbf{x} \in \mathbb{R}^{3} \backslash \bar{\Omega} .\end{cases}
$$

The electromagnetic field $\left(\mathbf{E}_{\eta}, \mathbf{H}_{\eta}\right)$ satisfies so-called Drude-Born-Fedorov equations within the chiral body:

$$
\left\{\begin{array}{l}
\nabla \times \mathbf{E}_{\eta}=i \omega \mu_{0} \mu_{\eta}\left(\mathbf{H}_{\eta}+\beta_{\eta} \nabla \times \mathbf{H}_{\eta}\right), \\
\nabla \times \mathbf{H}_{\eta}=-i \omega \varepsilon_{0} \varepsilon_{\eta}\left(\mathbf{E}_{\eta}+\beta_{\eta} \nabla \times \mathbf{E}_{\eta}\right),
\end{array}\right.
$$

where $\omega$ is the frequency, $\varepsilon_{0} \mu_{0}=c^{2}, c$ being the speed of light in vacuum. The ellipticity and boundedness of the operator are ensured by the following assumptions: $0<m \leq$ $\varepsilon_{r}, \mu_{r} \leq M$ and $0 \leq \beta<1$. The diffraction problem admits a unique solution under proper outgoing wave conditions $[2]$.

In fact, the smaller the typical heterogeneity size $\eta$, the faster the modulus of the electromagnetic field $\left(\mathbf{E}_{\eta}, \mathbf{H}_{\eta}\right)$ oscillates. Hence, we suppose that it can be approximated by a two-scale expansion of the form:

$$
\mathbf{E}_{\eta}(\mathbf{x})=\sum_{k=0}^{\infty} \eta^{k} \mathbf{E}_{k}\left(\mathbf{x}, \frac{\mathbf{x}}{\eta}\right), \mathbf{H}_{\eta}(\mathbf{x})=\sum_{k=0}^{\infty} \eta^{k} \mathbf{H}_{k}\left(\mathbf{x}, \frac{\mathbf{x}}{\eta}\right)
$$


where $\mathbf{E}_{k}, \mathbf{H}_{k}: \mathbb{R}^{3} \times Y \longmapsto \mathbb{C}^{3}$ are smooth functions of 6 variables, independent of $\eta$, such that $\forall \mathbf{x} \in \Omega, \mathbf{E}_{i}(\mathbf{x}, \cdot)$ and $\mathbf{H}_{i}(\mathbf{x}, \cdot)$ are $Y$-periodic.

Our goal is to characterize the diffracted field when $\eta$ tends to 0 .

\section{Homogenization result}

To identify the limit problem, we introduce the rescaled operator

$\nabla=\nabla_{x}+\frac{1}{\eta} \nabla_{y}$

where $x$ denotes the macroscopic (slow) variable and $y$ denotes the microscopic (fast) variable.

Substituting (2) and (3) in (1) and collecting the terms sitting in front of same powers of $\eta$ we obtain the following homogenized Drude-Born-Fedorov equations:

$$
\left\{\begin{aligned}
\nabla_{x} \times \mathbf{E}_{h o m} & =i \omega \mu_{0}\left[\mu_{h o m}\right]\left(\mathbf{H}_{h o m}\right. \\
& \left.+\left[\beta_{h o m}\right] \nabla_{x} \times \mathbf{H}_{h o m}\right) \\
\nabla_{x} \times \mathbf{H}_{h o m} & =-i \omega \varepsilon_{0}\left[\varepsilon_{h o m}\right]\left(\mathbf{E}_{h o m}\right. \\
& \left.+\left[\beta_{h o m}\right] \nabla_{x} \times \mathbf{E}_{h o m}\right) .
\end{aligned}\right.
$$

Similarly to the case of ferro-magnetic photonic crystals [3] (for which $\beta=0$ in (1)), the homogenized matrices $\left[\mu_{h o m}\right],\left[\varepsilon_{h o m}\right]$ are deduced from two auxiliary problems arising on the basic cell $Y$. But it turns out that they are now coupled and most importantly they do depend on the frequency $\omega$ of the incoming wave. Indeed, the auxiliary problem takes the following form:

$\nabla_{y} \cdot\left\{\left[M_{\beta, \omega}\right](y) \nabla_{y}\left(V_{\varepsilon}, W_{\mu}\right)\right\}=0$,

where

$\left[M_{\beta, \omega}\right](y)=\left(\begin{array}{ll}\frac{\varepsilon}{\Lambda} & \frac{i}{\omega} \sqrt{\frac{\varepsilon_{0}}{\mu_{0}}} \frac{1-\Lambda}{\beta \Lambda} \\ -\frac{i}{\omega} \frac{1-\Lambda}{\beta} \sqrt{\frac{\varepsilon_{0}}{\mu_{0}}} \frac{\mu}{\Lambda}\end{array}\right)$,

with

$\Lambda=1-\omega^{2} \beta^{2} \varepsilon \mu$.

We note that for ferro-magnetic media $\beta=0$, so that

$\Lambda=1$, and $(1-\Lambda) / \beta=\omega^{2} \beta \varepsilon \mu=0$.

Hence, one can see that off-diagonal entries of $\left[M_{\beta, \omega}\right]$ vanish in that case and we retrieve the result of [3].

We also note that

$$
\begin{aligned}
\operatorname{Det}\left(\left[M_{\beta, \omega}\right]\right) & =\sqrt{\frac{\varepsilon_{0}}{\mu_{0}}} / \Lambda^{2}\left(\varepsilon \mu-(1-\Lambda) / \omega^{2}\right) \\
& =\sqrt{\frac{\varepsilon_{0}}{\mu_{0}}} \varepsilon \mu\left(1-\beta^{2}\right) /\left(\omega^{2} \Lambda^{2}\right),
\end{aligned}
$$

which is positive since $0 \leq \beta<1$ (this is the case for chiral materials at hand [2]). Hence, from Lax-Milgram lemma there is a unique solution (up to an additive constant) to (5) in the space of periodic potentials of square integrable energy on $Y$.

The homogenized matrices of permittivity and permeability are given by

$$
\begin{aligned}
{\left[\varepsilon_{\text {hom }}\right] } & =\frac{<\varepsilon\left(I-\nabla_{y} V_{\varepsilon}\right)>}{<\Lambda>} \\
& -\frac{<\Lambda>}{\omega^{2}}<\frac{\Lambda-1}{\Lambda \beta}>^{2}<\mu\left(I-\nabla_{y} V_{\mu}\right)>^{-1}
\end{aligned}
$$

and

$$
\begin{aligned}
{\left[\mu_{\text {hom }}\right] } & =\frac{<\mu\left(I-\nabla_{y} V_{\mu}\right)>}{<\Lambda>} \\
& +\frac{<\Lambda>}{\omega^{2}}<\frac{\Lambda-1}{\Lambda \beta}><\varepsilon\left(I-\nabla_{y} V_{\varepsilon}\right)>^{-1}
\end{aligned}
$$

with $<.>=\int_{Y}$. $d y$. We remark that these matrices describe dispersive media (they depend upon $\omega$ ); Interestingly, if $\omega$ is small enough, $\left[\varepsilon_{h o m}\right]$ will become negative definite.

Finally, the homogenized matrix of chiral inductance can be expressed in two ways:

$$
\begin{aligned}
{\left[\beta_{\text {hom }}\right] } & =-\left[\xi_{\text {hom }}\right]^{-1} \frac{<\Lambda>}{\omega^{2}} \\
& <\frac{\Lambda-1}{\Lambda \beta}><\nu\left(I-\nabla_{y} V_{\nu}\right)>^{-1}
\end{aligned}
$$

where $(\xi, \nu) \in\{(\varepsilon, \mu),(\mu, \varepsilon)\}$. This makes an easily implementable consistency criterion to test convergence of a numerical algorithm.

\section{Conclusion}

The results obtained so far are only theoretical ones, but we are now investigating the numerical solutions of the auxiliary problems for realistic values of permittivity, permeability and chiral parameter thanks to finite elements modeling. We note that one can only prove a weak $L^{2}(\Omega)$ convergence of $\left(H_{\eta}, E_{\eta}\right)$ towards the leading order term $\left(H_{0}, E_{0}\right)$ of the asymptotic expansion (2). This is because $\left(H_{\eta}, E_{\eta}\right)$ is not divergence free, a fact which was already reported for the homogenization of ferro-magnetic photonic crystals in [3]. For a proof of strong $L^{2}(\Omega)$ convergence in the case of a dielectric (non-magnetic) photonic crystal, we refer the reader to [4].

\section{References}

[1] Pendry, J.B. Science 306, (2004) 1353.

[2] Ammari, H. and Bao, G. Matemat. Nach. 251, (2003) 3.

[3] Zolla, F. and Guenneau, S. Asymptotics, Singularities and Homogenisation in Problems of Mechanics, Ed. A.B. Movchan, Solid Mechanics and Its Applications SMIA, Chap. 6, p.375-385, Kluwer Academic Publishers, 2003 (ISBN: 1402017804).

[4] Guenneau, S. and Zolla, F. JEWA 14, (2000) 529. 